\title{
Electric dipole moments from postsphaleron baryogenesis
}

\author{
Nicole F. Bell, ${ }^{1, *}$ Tyler Corbett, ${ }^{1, \dagger}$ Michael Nee, ${ }^{1, *}$ and Michael J. Ramsey-Musolf ${ }^{2,}$ \\ ${ }^{1}$ ARC Centre of Excellence for Particle Physics at the Terascale School of Physics, \\ The University of Melbourne, Victoria 3010, Australia \\ ${ }^{2}$ Amherst Center for Fundamental Interactions, Department of Physics, \\ University of Massachusetts Amherst, Amherst, Massachusetts 01003, USA
}

(Received 26 September 2018; published 25 January 2019)

\begin{abstract}
We consider a model in which baryogenesis occurs at low scale, at a temperature below the electroweak phase transition. This model involves new diquark-type scalars which carry baryon number. Baryon number violation is introduced in the scalar potential, permitting $\Delta B=2$ violating process involving standard model quarks while avoiding stringent proton decay constraints. Depending on their quantum number assignment, the diquark-type scalars can couple to either right or left handed quarks, or to both. We show that this model can provide a viable explanation of the baryon asymmetry of the universe provided that the coupling to left handed quarks are present. However, the coexistence of couplings to left and right handed quarks introduces important phenomenological constraints on the model, such as radiative contributions to quark masses and the generation of electric dipole moments for nuclei, which probe the $C P$ even and $C P$ odd products of the relevant couplings constants, respectively. We demonstrate that the strongest such constraints arise from electric dipole moment measurements of the neutron and ${ }^{199} \mathrm{Hg}$. These constraints are sufficiently strong that, in the absence of an intricate flavor structure, baryogenesis must be dominated by the couplings of the new scalars to left handed quarks.
\end{abstract}

DOI: $10.1103 /$ PhysRevD.99.015034

\section{INTRODUCTION}

A major outstanding question in fundamental physics is the origin of the cosmological matter-antimatter asymmetry. While the necessary requirements to generate a baryon asymmetry are the well-known Sakharov conditions, namely (i) violation of baryon number (B), (ii) violation of $\mathrm{C}$ and $\mathrm{CP}$ symmetries (CPV) and (iii) departure from thermal equilibrium, the exact mechanism is yet to be established. Importantly, any mechanism that adequately fulfills these three requirements will require the introduction of new particles and interactions, beyond those that exist in the standard model.

A well studied mechanism is that of electroweak baryogenesis (EWBG) [1-4] where an asymmetry is generated via $C P$ violating interactions during a first order electroweak phase transition with the requisite $\mathrm{B}$ violation provided by electroweak sphalerons. The SM must be

\footnotetext{
n.bell@unimelb.edu.au

†corbett.t.s@gmail.com

*mnee@student.unimelb.edu.au

\$mjrm@physics.umass.edu
}

Published by the American Physical Society under the terms of the Creative Commons Attribution 4.0 International license. Further distribution of this work must maintain attribution to the author(s) and the published article's title, journal citation, and DOI. Funded by SCOAP ${ }^{3}$. augmented with new physics to make this scenario viable. An extended scalar sector is required to produce a strongly first order phase transition, which is necessary to fulfill the out-of-equilibrium requirement. In addition, new sources of CPV must be introduced as the SM Jarlskog factor is suppressed by small CKM matrix elements and small quark masses. These challenges notwithstanding, electroweak baryogenesis has the appealing feature of being a weakscale mechanism and thus eminently testable.

This is to be contrasted with high scale baryogenesis mechanisms, such as the out-of-equilibrium decay of a heavy particle. Leptogenesis [5-7] (type-I) is a particularly elegant example of such a scenario in which heavy singlet neutrinos-introduced to allow the seesaw mechanismdecay to lepton and antilepton final states with unequal rates. The resulting lepton asymmetry is subsequently converted to a baryon asymmetry via SM sphaleron processes. While the mechanism is very simple and requires minimal new physics, the energy scale is inaccessible to experiments, being $\gtrsim 10^{10} \mathrm{GeV}$ in the simplest scenarios.

In this paper we consider an out-of-equilibrium decay process which occurs at an experimentally accessible energy scale. In this sense, the mechanism shares the endearing features of both leptogenesis and EWBG. Notably, the asymmetry will be generated below the electroweak phase transition such that sphalerons play no role. The specific model we consider is a generalization of the "post-sphaleron 
baryogenesis" first introduced in Ref. [8], in which the B asymmetry is directly produced by the decays of a new scalar, $\Phi_{r}: \Phi_{r} \rightarrow 6 q$ and $\Phi_{r} \rightarrow 6 \bar{q}$. Importantly, the new physics in this model violates baryon number by two units, $\Delta B=2$. This renders the physics safe from the proton decay constraints that plague $\Delta B=1$ baryon number violating interactions, such as those in older GUT baryogenesis models. Because testable low energy baryogenesis mechanisms are relatively rare, this postsphaleron baryogenesis idea warrants further investigation, which is the purpose of this paper. Similar models have been studied in a nonbaryogenesis context in [9-12].

How do we experimentally test this scenario? The existence of $\Delta B=2$ baryon number violating processes can potentially be observed in neutron-antineutron oscillation measurements, as was considered in detail in Ref. [13]. A key focus in this paper will be to probe the $C P$ violating parameters of the model. A well established low energy technique to probe $C P$ violation is via the electric dipole moments (EDMs) of atoms, molecules, nucleons and nuclei, as these can be non-zero only in the presence of $C P$ violation (for recent reviews, see, e.g., Refs. [14-16]). We shall calculate EDMs together with naturalness constraints on radiative quark mass contributions, which place bounds on $C P$ odd and $C P$ even products, respectively, of the new coupling parameters in our model. These constraints will dictate which of the new couplings can play a role in baryogenesis.

A key difference between our work and that of Refs. [8,13,17] is the inclusion of couplings to both left handed (LH) and right handed (RH) quarks, rather than RH quarks alone. This has two significant consequences. First, because EDMs require a chirality flip, they will be generated at one loop only when couplings to LH and RH quarks are both present. Second, the size of the baryon asymmetry will depend on whether the interactions involve LH quarks, RH quarks, or both. Like all out-of-equilibrum decay scenarios, baryogenesis requires the interference of a tree level decay amplitude with the absorptive part of a one-loop amplitude. In postsphaleron baryogenesis, those loop diagrams involve $W^{ \pm}$gauge bosons and hence the distinction between couplings to LH or RH quarks is important. Indeed, we shall see that baryogenesis must be dominated by the couplings of the new scalars to LH quarks.

Our work is organized as follows: In Sec. II we outline the model and the postsphaleron baryogenesis mechanism. In Sec. III we compute constraints on the new coupling constants, using quark mass and nuclear EDM limits, while in Sec. IV we determine the dependence of the $C P$ asymmetries on those coupling parameters and hence discuss the implication of the constraints. We summarize our conclusions in Sec. V.

\section{THE MODEL}

We introduce three new colored scalar diquarks $\Delta_{d d}$, $\Delta_{u u}$, and $\Delta_{u d}$, which carry baryon number and couple to
TABLE I. The quantum number of the new scalars, together with their couplings to quark bilinears and the allowed quartic scalar interactions.

\begin{tabular}{lcccc}
\hline \hline Field & $S U(3)_{C}$ & $S U(2)_{L}$ & $U(1)_{Y}$ & Couplings \\
\hline$\Delta_{d d}$ & 6 & 1 & $-2 / 3$ & $d_{R} d_{R}$ \\
$\Delta_{u u}$ & 6 & 1 & $4 / 3$ & $u_{R} u_{R}$ \\
$\Delta_{u d}$ & 6 & 1 & $1 / 3$ & $u_{R} d_{R}$ \\
$\Phi$ & 1 & 1 & 0 & $\Delta_{d d} \Delta_{u d}^{2}, \Delta_{u u} \Delta_{d d}^{2}$ \\
\hline \hline
\end{tabular}

quark bilinears. Their quantum numbers and allowed couplings are outlined in Table I. In addition, we introduce a complex scalar field $\Phi$, which is a SM singlet, carries a baryon number of $B=2$, and has quartic interactions with the colored scalars of the form:

$$
V \supset \frac{\lambda}{2} \Phi \Delta_{d d} \Delta_{u d}^{2}+\frac{\lambda^{\prime}}{2} \Phi \Delta_{u u} \Delta_{d d}^{2}
$$

If the real part of $\Phi$, to which we will refer as $\Phi_{r}$, obtains a vacuum expectation value $\Delta B=2$ baryon number violating interactions are induced. Further details of the scalar potential have been discussed in $[8,13]$ and are relevant to the $N-\bar{N}$ oscillation calculations therein.

The interactions of SM fermions with the new colored scalars are given by

$$
\begin{aligned}
\mathcal{L}_{\text {Yukawa }}= & \frac{h_{\alpha \beta}}{2} \bar{K} \Delta_{d d}\left(\bar{d}_{R}\right)_{\alpha}\left(d_{R}\right)_{\beta}^{c} \\
& +\frac{f_{\alpha \beta}}{2} \bar{K} \Delta_{u u}\left(\bar{u}_{R}\right)_{\alpha}\left(u_{R}\right)_{\beta}^{c} \\
& +g_{\alpha \beta} \bar{K} \Delta_{u d}\left(\bar{u}_{R}\right)_{\alpha}\left(d_{R}\right)_{\beta}^{c} \\
& +g_{\alpha \beta}^{\prime} \bar{K} \Delta_{u d} \epsilon_{i j}\left(\bar{Q}_{i}\right)_{\alpha}\left(Q_{j}\right)_{\beta}^{c}+\text { H.c., }
\end{aligned}
$$

where $u_{R}$ and $d_{R}$ are the usual $\mathrm{RH}$ quark fields of hypercharge $Y=2 / 3$ and $-1 / 3$ respectively, while $Q$ is the LH quark doublet of hypercharge $Y=1 / 6$. The $\bar{K}$ matrices are the generators for $S U(3)_{c}$ in the sextet representation. Further details of the sextet representation may be found in [18]. We have used greek characters for flavor indices and the lower case latin characters $i, j$ for indices in the fundamental representation of $S U(2)_{L}$. We have and will continue to suppress color indices. They can be reinstated as follows:

$$
\bar{K}_{a b}^{A} \Delta^{A} \psi_{a} \chi_{b},
$$

where $A$ corresponds to a sextet color index, and $a, b$ correspond to indices in the fundamental representation. We note that the scalar diquarks are subject to strong constraints from the LHC. We shall adopt a benchmark value of $M_{\Delta}=10 \mathrm{TeV}$ throughout, which easily satisfies the current limits of $M_{\Delta} \gtrsim 6 \mathrm{TeV}$ [19]. 


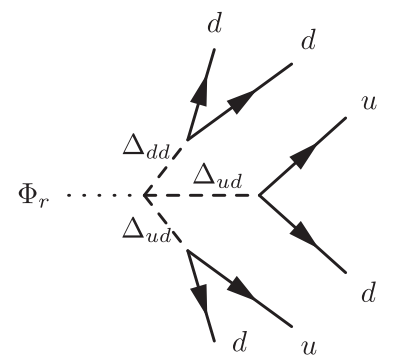

(a)

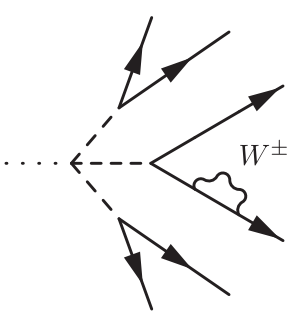

(b)

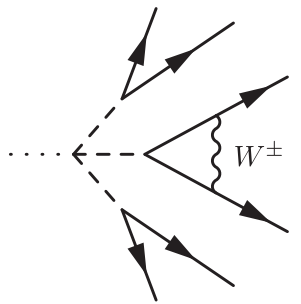

(c)

FIG. 1. Diagrams contributing to the $\Delta B=2$ decay of the real part of the scalar field $\Phi$ into six quarks. The dotted line corresponds to $\Phi_{r}$, the dashed lines to the new scalars $\Delta_{d d}, \Delta_{u u}$, and/or $\Delta_{u d}$, the solid lines are the final state quarks, and the one loop diagrams contain an intermediate $W$ boson. Diagram (a) corresponds to the tree level decay, (b) to the one loop self energy correction and (c) to the vertex correction diagram. Baryogenesis occurs via the interference of the tree diagram with the absorptive part of the loop diagrams. This requires that the $W$ in the loop is put on shell and hence a final state $t$-quark is necessary. If $\Phi_{r}$ is replaced with its vacuum expectation value (a) also corresponds to the diagram allowing $n-\bar{n}$ oscillations at tree level.

Notice that while $\Delta_{u u}$ and $\Delta_{d d}$ couple only to RH quarks, $\Delta_{u d}$ couples to both LH and RH quark bilinears. Although the $\Delta_{u d} Q Q$ coupling was not considered in the analyses of $[8,13]$, it cannot be forbidden without imposing additional symmetries. ${ }^{1}$ We find that the inclusion of this coupling to LH quarks has an important influence on both the EDM and baryogenesis phenomenology of the model.

Baryogenesis proceeds via the decay of the real part of the $\Phi$ field to six quarks or six antiquarks, thereby violating baryon number by two units. The Feynman diagram for the tree level decay is shown in Fig. 1(a). In order to obtain a nonzero baryon asymmetry we require the interference of the tree level decay amplitude with the absorptive part of the one loop decay amplitudes shown in Figs. 1(b) and 1(c). The first corresponds to corrections to the quark wave function via a $W$ exchange while the second corresponds to the vertex correction of the $\Delta_{u d}$ couplings $g$ or $g^{\prime}$. To obtain the absorptive part required for baryogenesis, we require the $W$ in the loop to be on shell; it is thus necessary to have a final state $t$-quark. We also note that of the three sextet scalars only two are required for successful baryogenesis: either $\left(\Delta_{d d}, \Delta_{u и}\right)$ or $\left(\Delta_{d d}, \Delta_{u d}\right)$ are sufficient, the latter being the focus of the studies $[8,13,17]$. As we are interested in the implications of including the left handed couplings of the $\Delta_{u d}$ boson we will also focus on the $\left(\Delta_{d d}, \Delta_{u d}\right)$ model throughout this paper.

If the dotted line in Fig. 1(a) is replaced with the vacuum expectation value of $\Phi_{r}$, the resulting diagram describes a mechanism for neutron-antineutron oscillations. This both puts constraints on the relevant couplings of the model as well as motivating future studies of neutron oscillations [13].

As we shall be interested in low energy observables and in baryogenesis that takes place below the electroweak scale, we choose to work in the quark mass basis. Rotating

\footnotetext{
${ }^{1}$ A UV completion based on a left-right symmetric model eliminates this coupling in $[8,13]$.
}

to the mass basis results in a redefinition of the couplings of Eq. (2), as detailed in Appendix A. The resulting Lagrangian coupling up and down type quarks to $\Delta_{u d}$ is

$\mathcal{L}=\bar{K} \Delta_{u d}\left(\bar{u}_{R}^{\prime}\right) G\left(d_{R}^{\prime}\right)^{c}+2 \bar{K} \Delta_{u d}\left(\bar{u}_{L}^{\prime}\right) G^{\prime}\left(d_{L}^{\prime}\right)^{c}+$ H.c..

Capital $G$ and $G^{\prime}$ are the mass basis analogues of the couplings in Eq. (2), and the generation indices have been suppressed for simplicity. Although we perform our calculations in the mass basis, we will still reference the properties of the couplings in the flavor basis to derive certain results in Sec. IV as the flavor structure is crucial in identifying the leading contributions to the baryon asymmetry. We are now in a position to calculate constraints on the $G$ and $G^{\prime}$ matrices and the implications of those constraints on the parameter space for successful baryogenesis.

\section{QUARK MASS AND EDM CONSTRAINTS}

As mentioned above, the introduction of the new LH coupling of the $\Delta_{u d}$ has the potential make significant new contributions to the baryon asymmetry. An important question to address, however, is whether the coexistence of LH and RH couplings will imply new low energy constraints which might render the new contributions to the baryon asymmetry negligibly small. Of particular concern are operators that break chiral symmetry: the standard model Yukawa interactions and dipole operators. Both will in general receive new radiative contributions with the simultaneous presence of both LH and RH $\Delta_{u d}$ couplings. In the case of Yukawa interactions, "naturalness" The strongest constraints are associated with intermediate considerations imply that radiative corrections to the Yukawa couplings should not be considerably larger in magnitude than their tree-level values. With regard to dipole operators, experimental limits on the EDMs of the neutron and ${ }^{199} \mathrm{Hg}$ atom imply severe restrictions on the relative phases of the $G$ and $G^{\prime}$ couplings. In what follows, we analyze both considerations in detail. In doing so, we will work 


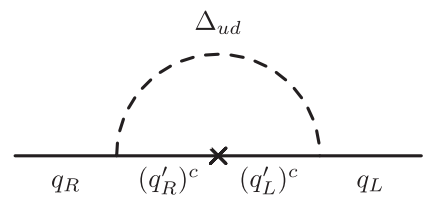

FIG. 2. One-loop diagrams contributing to the radiatively generated quark mass. The intermediate quark mass insertion is marked by $\times$ and the dominant contributions come from heavy quarks in the loop. Only $\Delta_{u d}$, which couples to both LH and RH quarks, can give rise to such mass contributions; there are no analogous diagrams containing $\Delta_{u u}$ or $\Delta_{d d}$.

with quantities and the degrees of freedom associated with the standard model after electroweak symmetrybreaking: quark masses and quark (chromo-)electric dipole moments. ${ }^{2}$

\section{A. Radiative quark masses and naturalness}

The couplings of the quarks to the scalar $\Delta_{u d}$ give rise to radiative quark mass contributions, as shown in Fig. 2. We shall impose a naturalness constraint on the size of these mass contributions, by requiring that the radiatively generated contribution be much smaller in magnitude than the physical quark mass.

Calculating the one loop generated up quark mass at the quark mass scale after running down from the new physics (NP) scale and requiring it be smaller than the tree level quark mass gives the constraint:

$$
\delta m_{j}^{u}=\frac{4 N_{c} m_{i}^{d}}{8 \pi^{2}}\left|G_{i j}^{*} G_{i j}^{\prime}\right| \log \frac{\left(m_{j}^{u}\right)^{2}}{M_{\Delta_{u d}}^{2}} \ll m_{j}^{u} .
$$

The constraint for the down quark mass comes from replacing the labels $u \leftrightarrow d$ and changing the order of the indices of $G$ and $G^{\prime}$. Note there is an implied sum over flavors $i$, but not $j$. We assume that phases in the quark mass matrix, equivalent to a QCD theta term, are removed by assumption of a Pecci-Quinn symmetry. Our constraints thus apply to the magnitude of the radiative mass contributions. Here we have used the benchmark value $M_{\Delta_{u d}}=10 \mathrm{TeV}$, which easily satisfies LHC constraints on the diquark masses [19].

The strongest constraints are associated with intermediate heavy quarks $(i=3)$, leading to

$$
\begin{aligned}
& \left|G_{13}^{*} G_{13}^{\prime}\right|<10^{-5}, \\
& \left|G_{31}^{*} G_{31}^{\prime}\right|<10^{-4} .
\end{aligned}
$$

\footnotetext{
${ }^{2}$ The resulting constraints are equivalent to those that one would obtain by first considering the SM in its electroweak symmetric phase and then applying the results to the broken phase quantities.
}

Next we consider the EDM constraints which, in contrast with the mass, are sensitive to the imaginary part of the same products of $G^{*}$ and $G^{\prime}$ elements.

\section{B. Electric dipole moments framework}

In studying the constraints from EDM measurements we will closely follow the notation of [15]. We quantify the NP contributions to the EDMs in terms of dimension six operators by defining the effective Lagrangian, ${ }^{3}$

$$
\mathcal{L}_{\text {eff }}=\sum_{i} \frac{c_{i}}{\Lambda^{2}} Q_{i}+\mathcal{O}\left(\Lambda^{-3}\right),
$$

where the operators $Q_{i}$ are constructed from SM fields, and $c_{i}$ are their Wilson coefficients. The operators admitting $C P$ violation at dimension-six include 10 operators coupling fermions to bosons as well as five four-fermion operators which are enumerated in Tables 2 and 3 of [15]. Here we list only those relevant to our analysis,

$$
\begin{gathered}
Q_{q G}=\left(\bar{Q} \sigma^{\mu \nu} T^{A} q_{R}\right) \stackrel{(\sim)}{H} G_{\mu \nu}^{A}, \\
Q_{q W}=\left(\bar{Q} \sigma^{\mu \nu} q_{R}\right) \tau^{i} \stackrel{(\sim)}{H} W_{\mu \nu}^{i}, \\
Q_{q B}=\left(\bar{Q} \sigma^{\mu \nu} q_{R}\right) \stackrel{(\sim)}{H} B_{\mu \nu}, \\
Q_{q u q d}^{(1)}=\left(\bar{Q}^{i} u_{R}\right) \epsilon_{i j}\left(\bar{Q}^{j} d_{R}\right), \\
Q_{q u q d}^{(8)}=\left(\bar{Q}^{i} T^{A} u_{R}\right) \epsilon_{i j}\left(\bar{Q}^{j} T^{A} d_{R}\right) .
\end{gathered}
$$

where $G^{A \mu \nu}$ the gluon field strength tensor with adjoint color index $A, W^{i \mu \nu}$ is the $S U(2)$ field strength tensor with adjoint $S U(2)_{L}$ index $i$, and $B^{\mu \nu}$ is the hypercharge field strength tensor. We denote the SM Higgs doublet as $H$, and define $\tilde{H}=i \sigma_{2} H$ where $\sigma_{2}$ the second Pauli matrix. Here, $q_{R}$ represents either $u_{R}$ or $d_{R}$ and is associated with the $\tilde{H}$ or $H$, respectively. After electroweak symmetry breaking, wherein

$$
H^{T} \rightarrow(0, v / \sqrt{2}),
$$

the operators in Eqs. (9) and (10) generate the dipole couplings to the photon, $Z$, and $W$ bosons. For example, one obtains the effective EDM interaction

$$
\mathcal{L}_{q \gamma}^{\mathrm{EDM}}=i \frac{\sqrt{2} v}{2} \frac{\operatorname{Im}\left[c_{q \gamma}\right]}{\Lambda^{2}} \bar{q}_{L} \sigma^{\mu \nu} \gamma_{5} q F_{\mu \nu},
$$

where $F_{\mu \nu}$ is the field strength tensor of the photon field and we have chosen the normalization of the operator coefficient to coincide with that of [15]:

\footnotetext{
${ }^{3}$ Note that we do not explicitly include the dimension-five lepton-number violating operator.
} 


$$
\operatorname{Im}\left[c_{q \gamma}\right]=\operatorname{Im}\left[c_{q B}\right]+I_{3}^{q} \operatorname{Im}\left[c_{q W}\right]
$$

with $I_{3}^{q}$ being the third component of weak isospin for quark $q$. Note that in the notation of Ref. [15] the coefficients $c_{q B}$ and $c_{q W}$ enter the effective Lagrangian with explicit factors of the $\mathrm{U}(1)_{Y}$ and $\mathrm{SU}(2)_{L}$ gauge couplings, respectively, so that the relation in Eq. (15) carries no explicit dependence on the weak mixing angle.

After integrating out the heavy $\Delta_{u d}$ scalar, we find that the four fermion operators $Q_{q u q d}^{(1)}$ and $Q_{q u q d}^{(8)}$ are generated at tree-level, while the dipole operators $Q_{f W}$ and $Q_{f B}$ and the chromo-dipole operators $Q_{u G}$ and $Q_{d G}$ arise at one-loop. The four-quark and (chromo-)EDM operators, in turn, contribute to the EDM of the neutron, while only the four-quark and chromo-EDM operators generate potentially significant contributions to the ${ }^{199} \mathrm{Hg}$ atomic EDM via the nuclear Schiff moment as outlined in [15]. In the next two subsections, we first derive the relevant operator Wilson coefficients. We then outline the procedure for deriving the implications for the neutron and ${ }^{199} \mathrm{Hg}$ EDMs and finally apply EDM constraints to the relevant combinations of the $G$ and $G^{\prime}$ couplings.

\section{EDM and chromo-EDM operators at the partonic scale}

In principle, since both the four-quark and (chromo-) EDM operators arise in our scenario, one must consider contributions from both to the EDMs of diamagnetic systems. In practice, consideration of the four-quark contribution is not presently tractable, due to particularities of the model considered here. In general, the operators $Q_{\text {quqd }}^{(1,8)}$ containing only first generation quarks admit an $\mathrm{SU}(2)_{L} \times$ $\mathrm{SU}(2)_{R}$ chiral symmetry. Based on this feature, one is able to obtain order-of-magnitude estimates of the hadronic matrix elements relevant to EDMs using naïve dimensional analysis (NDA), even in the absence of explicit lattice QCD or model computations.

As discussed in Appendix B, however, in the basis of weak interaction (flavor) eigenstates, the Wilson coefficients of $Q_{\text {quqd }}^{(1,8)}$ vanish for first generation quarks. This result follows from the antisymmetry of the $g^{\prime}$ couplings with respect to flavor indices. Nonvanishing contributions arise when the flavor of the two RH quarks differs. After rotating to the quark mass basis, one obtains effective fourquark operators involving all first generation quarks as well as those involving other quark flavors. However, operators containing only first generation quarks no longer reflect the $\mathrm{SU}(2)_{L} \times \mathrm{SU}(2)_{R}$ chiral symmetry associated with $Q_{q u q d}^{(1,8)}$ in the flavor basis, since the operators in the flavor basis also do not reflect this symmetry in our model. Consequently, we are not presently able to estimate the hadronic matrix elements using NDA and considerations of chiral symmetry, as was done in Ref. [15]. Instead, we
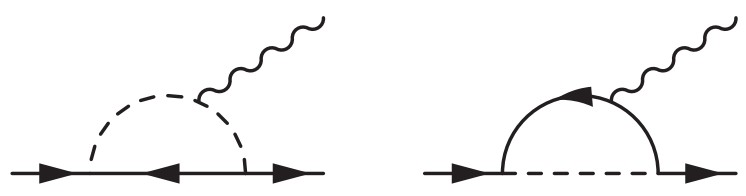

FIG. 3. One-loop diagrams contributing to the up- and down-quark EDMs. The dashed lines represent the new scalar $\Delta_{u d}$, the external solid lines represent either the up- or downquark, while the intermediate solid line is summed over all active flavors of quarks. Left: the new heavy scalar $\Delta_{u d}$ radiates a photon or gluon, Right: the intermediate quark radiates a photon or gluon.

focus on the loop-induced (chromo-)EDM contributions, using them to obtain conservative upper bounds on the relevant combinations of the mass basis $G$ and $G^{\prime}$ couplings. Any significant additional contributions from the four quark $\mathrm{CPV}$ operators would only strengthen the bounds quoted below in the absence of finely-tuned cancellations among the various contributions.

The quark (chromo-)EDMs and chromo EDMs receive one-loop contributions from the diagrams shown in Fig. 3 (We note that similar derivation for the $d$-quark EDM alone can be found in [9]). We generate the Feynman rules for our model with the FeYnRules Mathematica package [20], calculate the amplitudes in FEYNARTS [21] and FORMCALC [22], and finally obtain analytic forms for the amplitudes along with their leading order terms in the momentum expansion using PACKAGE-X [23].

We obtain

$\operatorname{Im}\left[c_{u \gamma}\right]=-\frac{m_{\alpha}}{12 \pi^{2} \sqrt{2} v} \operatorname{Im}\left[G_{\alpha 1}^{\prime} G_{\alpha 1}^{*}\right]\left[4+2 \ln \left(\frac{m_{\alpha}^{2}}{M_{\Delta_{u d}}^{2}}\right)\right]$,

$\operatorname{Im}\left[c_{d \gamma}\right]=\frac{m_{\alpha}}{6 \pi^{2} \sqrt{2} v} \operatorname{Im}\left[G_{1 \alpha}^{\prime} G_{1 \alpha}^{*}\right]\left[\frac{5}{2}+2 \ln \left(\frac{m_{\alpha}^{2}}{M_{\Delta_{u d}}^{2}}\right)\right]$,

where $\alpha$ corresponds to a down-type quark in Eq. (16) and an up-type quark in Eq. (17). We note that for $\alpha=3$ these operators have a strong enhancement from the top and bottom quark masses. We will discuss this feature in more detail below.

The computation of the chromo-EDM is similar, replacing the external photon with a gluon. We find the only difference between the up- and down-quark chromo-EDMs arises from the difference in mass for each generation and from the couplings $G$ and $G^{\prime}$. Using a similar normalization as in Eq. (7) we obtain

$$
\begin{aligned}
\operatorname{Im}\left[c_{u G}\right] & =\frac{m_{\alpha}}{8 \pi^{2} \sqrt{2} v} \operatorname{Im}\left[G_{\alpha 1}^{\prime} G_{\alpha 1}^{*}\right] \ln \left(\frac{m_{\alpha}^{2}}{M_{\Delta_{u d}}^{2}}\right), \\
\operatorname{Im}\left[c_{d G}\right] & =\frac{m_{\alpha}}{8 \pi^{2} \sqrt{2} v} \operatorname{Im}\left[G_{1 \alpha}^{\prime} G_{1 \alpha}^{*}\right] \ln \left(\frac{m_{\alpha}^{2}}{M_{\Delta_{u d}}^{2}}\right) .
\end{aligned}
$$


In our analysis below we will make the simplifying assumption that all elements of $G$ and $G^{\prime}$ are of the same order. Under this assumption we see that the largest contribution to the up and down quark (chromo) EDMs come from the diagrams with bottom and top quarks in the loops, respectively, due to the dependence on the $m_{\alpha}$. As a result, we expect the limits arising from the $d$-quark EDM to be stronger that those for the $u$-quark EDM by a factor of about $\operatorname{Im}\left[c_{d G}\right] / \operatorname{Im}\left[c_{u G}\right] \sim m_{t} / m_{b} \sim 40$. This ratio will be observed in the bounds arising from neutron and ${ }^{199} \mathrm{Hg}$ EDM constraints.

\section{EDMs of the neutron and ${ }^{199} \mathrm{Hg}$}

We now calculate the new contributions to the neutron and ${ }^{199} \mathrm{Hg}$ EDMs using the operator coefficients determined above. In order to do so we must evolve the coefficients from the heavy scale specified by $M_{\Delta_{u d}}$ to the hadronic scale, which we take to be one GeV. We obtain the operator coefficients at this scale using the renormalization group analysis of [24], wherein one first evolves down to EW scale integrating out the $t$-quark along the way. Matching is performed while integrating out electroweak gauge bosons and they evolve down to the hadronic scale integrating out the $b$ and $c$ quarks along the way.

We calculate the neutron $\mathrm{EDM}, d_{n}$, following the procedure of [15]:

$$
d_{n}=\sum_{q=u, d} \frac{v^{2}}{M_{\Delta_{u d}}^{2}}\left(\beta_{n}^{q \gamma} \operatorname{Im}\left[c_{q \gamma}\right]+\beta_{n}^{q G} \operatorname{Im}\left[c_{u G}\right]\right)
$$

where the $\operatorname{Im}\left[c_{q \gamma}\right]$ are evaluated at the scale $\mu=1 \mathrm{GeV}$ and where the hadronic matrix elements are encoded in the $\beta_{i}^{j}$. We reproduce their values in Table II for convenience. Note that the values of the $\beta_{n}^{q \gamma}$ have been updated to reflect the recent lattice QCD computations of Refs. [25,26]. Table II also gives ranges for the $\beta_{i}^{j}$ etc., to illustrate their degree of uncertainty, however we will use only the central values in our analysis. As pointed out in [25,26], one may also anticipate non-negligible contributions from the strange quark EDM, though the magnitude of this contribution is rather uncertain. We have not included this uncertainty in Table II.

To calculate the EDM of ${ }^{199} \mathrm{Hg}$, we begin with the isoscalar and isovector coefficients of the $T$ and $P$-violating pion-nucleon Lagrangian, $\bar{g}_{\pi}^{(0)}$ and $\bar{g}_{\pi}^{(1)}$ respectively, expressed in terms of our effective operator coefficients:

$$
\bar{g}_{\pi}^{(i)}=\frac{v^{2}}{M_{\Delta_{u d}}^{2}} \gamma_{(i)}^{ \pm G}\left(\operatorname{Im}\left[c_{u G}\right] \pm \operatorname{Im}\left[c_{d G}\right]\right)
$$

where the $\gamma_{i}$ are given in Table II. The isotensor contribution, $\bar{g}_{\pi}^{(2)}$, is a subleading effect and is neglected in our analysis. The dipole moment of ${ }^{199} \mathrm{Hg}$ is then given by
TABLE II. Best values and ranges for the coefficients relevant to the neutron and ${ }^{199} \mathrm{Hg}$ EDM calculations, all values were taken from [15] except the updated values for $\beta_{n}^{q \gamma}$ were taken from $[25,26]$.

\begin{tabular}{lcc}
\hline \hline Coefficient & Best value [e fm] & Range [e fm] \\
\hline$\beta_{n}^{u G}$ & $4 \times 10^{-4}$ & $(1,10) \times 10^{-4}$ \\
$\beta_{n}^{d G}$ & $8 \times 10^{-4}$ & $(2,18) \times 10^{-4}$ \\
$\beta_{n}^{u \gamma}$ & $1.3 \times 10^{-3}$ & $(1.1,1.5) \times 10^{-3}$ \\
$\beta_{n}^{d \gamma}$ & $-4.4 \times 10^{-3}$ & $-(4.1,4.7) \times 10^{-3}$ \\
$\beta_{n}^{q u q d}$ & $40 \times 10^{-7}$ & $(10,80) \times 10^{-7}$ \\
$\gamma_{(0)}^{+G}$ & -0.01 & $(-0.03,0.03)$ \\
$\gamma_{(1)}^{-G}$ & -0.02 & $-(0.07,0.01)$ \\
$\gamma_{(1)}^{q u q d}$ & $2 \times 10^{-6}$ & $(1,10) \times 10^{-6}$ \\
\hline \hline
\end{tabular}

$$
d_{A}=\rho_{p} d_{p}+\rho_{n} d_{n}+\frac{2 m_{N} g_{A} \kappa_{S}}{F_{\pi}}\left(a_{0} \bar{g}_{\pi}^{(0)}+a_{1} \bar{g}_{\pi}^{(1)}\right),
$$

where $d_{p}$, the proton EDM, is calculated using Eq. (20) with $\left(\beta_{n}^{u \gamma} \leftrightarrow \beta_{n}^{d \gamma}\right)$ and $\left(\beta_{n}^{u G} \leftrightarrow \beta_{n}^{d G}\right)$, i.e., by invoking isospin invariance. The last term in the expression above is the contribution from the nuclear Schiff moment. Values assumed for the nucleon mass $m_{N}$, the leading order strong interaction coupling $-2 g_{A} / F_{\pi}$, pion decay constant $F_{\pi}$, and the coefficients $a_{i}$ can be found in Table III.

The current 95\% confidence limits on the EDMs of the neutron [27] and ${ }^{199} \mathrm{Hg}$ [28] are

$$
\begin{gathered}
\left|d_{\mathrm{n}}\right| \leq 3.6 \times 10^{-13} \text { e fm }, \\
\left|d_{\mathrm{Hg}}\right| \leq 7.4 \times 10^{-17} \text { e fm } .
\end{gathered}
$$

By demanding that the new physics contributions to these parameter are smaller than the experimental bounds, we will set limits on the $G$ and $G^{\prime}$ coupling constants. In order to do so, however, we will need to make some simplifying assumptions about the structure of the coupling matrices. ${ }^{4}$ We shall assume that all elements of $G$ are of the same order of magnitude, and make a similar assumption about the elements of $G^{\prime}$. Note, however, that we permit the overall scales of $G$ and $G^{\prime}$ to differ significantly. Given this assumption and that $\operatorname{Im}\left[c_{q \gamma, g}\right]$ are all proportional to $m_{\alpha}$ [see Eqs. (16) through (19)] we see that the terms enhanced

\footnotetext{
${ }^{4}$ The matrix $G$ has 9 complex parameters, in general, while and $G^{\prime}$ has the three complex parameters arising from $g^{\prime}$ plus the four CKM parameters (see the definition of $G^{\prime}$ in Appendix A). To individually constrain all elements of these matrices would require a significant phenomenological effort, well beyond the scope of this paper. We will therefore make convenient simplifying assumptions about the structure of the matrices. More complex flavor structures are possible, such as those considered in $[8,13]$.
} 
TABLE III. Parameters values used in the EDM analysis, taken from Tables 4 and 13 of [15]. Note that the entry for $\kappa_{S}$ corrects the overall sign of this quantity from what is given in Ref. [15].

\begin{tabular}{lcc}
\hline \hline Parameter & Value & Range \\
\hline$m_{N}$ & $938 \mathrm{MeV}$ & $\ldots$ \\
$g_{A}$ & 1.33 & $\ldots$ \\
$F_{\pi}$ & $185 \mathrm{MeV}$ & $\ldots$ \\
$a_{0}$ & 0.01 & $(0.005,0.05)$ \\
$a_{1}$ & \pm 0.02 & $(-0.03,0.09)$ \\
$a_{2}$ & 0.02 & $(0.01,0.06)$ \\
$\rho_{p}$ & $-0.56 \times 10^{-4}$ & $\ldots$ \\
$\rho_{n}$ & $-5.3 \times 10^{-4}$ & $\ldots$ \\
$\kappa_{S}$ & $-2.8 \times 10^{-4} \mathrm{fm}^{-2}$ & $\cdots$ \\
\hline \hline
\end{tabular}

by the heavy quark masses will dominate the limits. Therefore, the EDM constraint directly probe the coupling combinations $\operatorname{Im}\left[G_{13}^{\prime} G_{13}^{*}\right]$ and $\operatorname{Im}\left[G_{31}^{\prime} G_{13}^{*}\right]$.

Figure 4 shows the region in the $\operatorname{Im}\left[G_{13}^{\prime} G_{13}^{*}\right]$ vs $\operatorname{Im}\left[G_{31}^{\prime} G_{31}^{*}\right]$ plane in which the $95 \%$ confidence level limits of $d_{N}$ and $d_{\mathrm{Hg}}$ are not exceeded. We neglect to perform a more formal likelihood analysis here as we expect our discussion of the baryon asymmetry in Sec. IV only to be an order of magnitude estimate. Parameters which pass the neutron EDM constraint are shown in red, while those which pass the ${ }^{199} \mathrm{Hg}$ constraint are shown in blue. The allowed parameter space is hence given roughly by the region where these bands intersect. Where we have again taken a benchmark value for $M_{\Delta_{u d}}$ of $10 \mathrm{TeV}$. For different values of $M_{\Delta_{u d}}$ the results scale approximately as $[\mathrm{TeV}]^{2} / M_{\Delta_{u d}}^{2}$; this naive scaling rule is broken only by

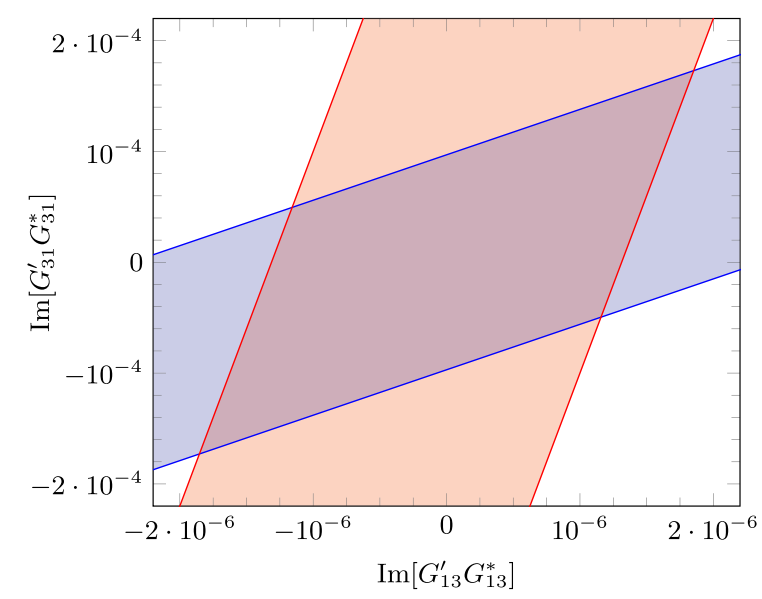

FIG. 4. The blue region is allowed by the ${ }^{199} \mathrm{Hg}$ EDM constraints, while the red region is allowed by the neutron EDM constraints. The region allowed by both constraints is shown as purple. We have taken $M_{\Delta_{u d}}=10 \mathrm{TeV}$ in order to comply with the constraints on diquark masses from the LHC [19]. For different values of $M_{\Delta_{u d}}$ the results scale roughly as $[\mathrm{TeV}]^{2} / M_{\Delta_{u d}}^{2}$. the logarithms in the one loop Wilson coefficients of Eqs. (16)-(19) and by the running of the effective couplings from the $M_{\Delta_{u d}}$ scale to $M_{\mathrm{QCD}}=1 \mathrm{GeV}$.

We find that

$$
\begin{aligned}
\left|\operatorname{Im}\left[G_{13}^{\prime} G_{13}^{*}\right]\right| & <1.9 \times 10^{-6}, \\
\left|\operatorname{Im}\left[G_{31}^{\prime} G_{31}^{*}\right]\right| & <1.7 \times 10^{-4} .
\end{aligned}
$$

As mentioned above, the difference in the constraints simply reflects the ratio $m_{t} / m_{b} \sim 40$. For $G_{i j} \sim G_{i j}^{\prime}$, these bounds conservatively correspond to $G$ and $G^{\prime}$ elements of order $10^{-3}$. The constraints coming from the EDMs and those from the radiative quark masses are complementary as they constrain the imaginary part and magnitude, respectively, of $\left(G^{\dagger} m_{u, d} G^{\prime}\right)_{i i}$. From the results presented in Eqs. (6) and (25), we conclude that the imaginary parts of these quantities are small, while the real parts can be an order of magnitude or more larger. When we come to the baryogenesis analysis, however, we will simply assume that both the real and imaginary components (and hence the magnitudes) of the $G G^{\prime}$ quantities satisfy the more stringent of these bounds [Eq. (25)], while the phase is unconstrained, as this will lead to the maximum baryon asymmetry. ${ }^{5}$

In the next section we will use these constraints to analyze three main benchmark scenarios for baryon asymmetry generation. These benchmarks are specified by $\Delta_{u d}$ couplings to only RH quarks, only to LH quarks, or to both, namely:

$$
\begin{aligned}
& G \sim 1 \quad \text { and } \quad G^{\prime} \sim 0, \\
& G \sim 0 \quad \text { and } \quad G^{\prime} \sim 1, \\
& G \sim 10^{-3} \quad \text { and } \quad G^{\prime} \sim 10^{-3} .
\end{aligned}
$$

The last benchmark corresponds to $G G^{\prime} \sim 10^{-6}$, which is the most conservative assumption give the EDM bounds derived above and easily satisfies the radiative mass bounds.

\section{BARYOGENESIS}

\section{A. $C P$ asymmetry of the $\Phi$ decays}

The baryon asymmetry is generated at temperatures below the EW phase transition via the out-of-equilibrium decays of $\Phi_{r} \rightarrow 6 q$ and $\Phi_{r} \rightarrow 6 \bar{q}$, shown in Fig. 1. The requisite $C P$ violation is provided by the complex $G$ and $G^{\prime}$ coupling constants, and through the interference of the tree level decay amplitude with the absorptive part of the 1-loop amplitudes. A key requirement is the presence of $W$ gauge bosons in the loop graphs because, as we shall see below,

\footnotetext{
${ }^{5}$ The $C P$-violating asymmetry in $\Phi$ decays is proportional to the imaginary part of a product of couplings, divided by a real part (arising from the tree level decay rate).
} 
the non-diagonal flavor structure of the CKM matrix is necessary to generate a nonzero asymmetry. For this reason, there is no analogous $Z$ boson contribution.

As mentioned above, our model differs from that examined in Refs. $[8,13,17]$ by the addition of the $G^{\prime}$ couplings of $\Delta_{u d}$ to LH quarks. We expect this to allow larger baryon asymmetries to be generated, because the loop diagrams that permit baryogenesis all involve $W$ bosons. Specifically, if the scalar $\Phi_{r}$ decays to only RH quarks, then chiral flips are required to permit couplings of the quarks to the $W$, and hence we expect the rates to be suppressed by factors of quark mass. However, if $\Phi_{r}$ couples to LH quarks, no chiral flip is needed. We therefore expect the baryon asymmetry generated via processes involving $\mathrm{RH}$ quarks to be suppressed by factors of $m_{\text {light quark }} / M_{\Phi}$ with respect to the asymmetry generated by processes involving LH quarks.

A full calculation of the baryon asymmetry is beyond the scope of this work. Such a calculation would involve an evaluation of the Boltzmann equations required to track the evolution of the asymmetry, accounting for the $\Phi_{r}$ decays together with any relevant dilution or washout effects. We defer such a calculation, together with a detailed exploration of the parameter space, to a future publication. Instead, as in Refs. $[8,13,17]$, we determine the $C P$ asymmetry of the decay rates, defined as

$$
\epsilon=\frac{\Gamma(\Phi \rightarrow 6 q)-\Gamma(\Phi \rightarrow 6 \bar{q})}{\Gamma(\Phi \rightarrow 6 q)+\Gamma(\Phi \rightarrow 6 \bar{q})} .
$$

The $C P$ asymmetry represents an upper bound on the net baryon asymmetry. In practice, the final baryon asymmetry will be reduced somewhat by dilution or washout effects.

The total $\Phi_{r}$ decay rate at tree level is given by:

$$
\begin{aligned}
\Gamma_{\text {tree }}^{\mathrm{TOT}} & \equiv \Gamma(\Phi \rightarrow 6 q)+\left.\Gamma(\Phi \rightarrow 6 \bar{q})\right|_{\text {tree }} \\
& =\frac{1}{\pi^{9} \cdot 2^{25} \cdot 45} 12|\lambda|^{2} \operatorname{Tr}\left[H^{\dagger} H\right] \frac{M_{\Phi}^{13}}{M_{\Delta_{u d}}^{8} M_{\Delta_{d d}}^{4}} X^{2},
\end{aligned}
$$

where the phase space factor $1 /\left(45 \cdot 2^{25} \pi^{9}\right)$ has been factored out of the six body phase space [29], and the factor 12 is a color factor. We have defined the quantity $\boldsymbol{X}$, which contains the dependence on the $G$ and $G^{\prime}$ couplings constants, as

$$
\begin{aligned}
\boldsymbol{X} \equiv & \operatorname{Tr}\left[G^{\dagger} G+\left(G^{\prime}\right)^{\dagger} G^{\prime}\right] P_{1} \\
& -\operatorname{Tr}\left[\left(G^{\prime}\right)^{\dagger} \boldsymbol{m}_{u} G \boldsymbol{m}_{d}+G^{\dagger} \boldsymbol{m} v_{u} G^{\prime} \boldsymbol{m}_{d}\right] \frac{P_{0}}{M_{\Phi}^{2}} .
\end{aligned}
$$

Here and below $\boldsymbol{m}_{u, d}$ are the $3 \times 3$ diagonal up/down quark mass matrices. The quantities $P_{1}$ and $P_{0}$ arise from the six body phase space integral. Terms weighted by $P_{1}\left(P_{0}\right)$ are proportional to phase space integrals of $p_{i} \cdot p_{j}\left(m_{i} m_{j}\right)$. Following [13] we simplify the momentum dependent integral by assuming the momentum is averaged over the six final state quarks yielding $p_{i} \cdot p_{j} \sim M_{\Phi}^{2} / 6$ and $P_{0} \sim P_{1} / 6$. We use $P_{1} \sim 10^{-4}$, obtained by numerical integration of the 6-body phase space.

There are two dominant contributions to the baryon asymmetry. The first from interference of the tree level decay amplitude with the $t$-quark self energy diagram of Fig. 1(b), and the second from the interference with the vertex correction of Fig. 1(c). In both cases a final state $t$-quark is required so that the $W$ boson can be put on shell, which is necessary for obtaining an absorptive part. Evaluating the wave function and vertex contributions to the asymmetries, we find

$$
\begin{aligned}
\epsilon_{\mathrm{wave}} \sim & \frac{3 g_{w}^{2}}{32 \pi \boldsymbol{X} \boldsymbol{M}_{W}^{2} m_{t}^{2}} \operatorname{Im}\left(\left[V \boldsymbol{m}_{d}^{2} V^{\dagger} \boldsymbol{m}_{u} G G^{\dagger} \boldsymbol{m}_{u}\right]_{33} P_{1}\right. \\
& +m_{t}^{2}\left[V \boldsymbol{m}_{d}^{2} V^{\dagger} G^{\prime} G^{\prime \dagger}\right]_{33} P_{1} \\
& \left.-m_{t}^{2}\left[V \boldsymbol{m}_{d}^{2} V^{\dagger}\left(\boldsymbol{m}_{u} G \boldsymbol{m}_{d} G^{\prime \dagger}+G^{\prime} \boldsymbol{m}_{d} G^{\dagger} \boldsymbol{m}_{u}\right)\right]_{33} \frac{P_{0}}{M_{\Phi}^{2}}\right),
\end{aligned}
$$

$$
\begin{aligned}
\epsilon_{\mathrm{vertex}} \sim & \frac{g_{w}^{2}}{32 \pi \boldsymbol{X} M_{W}^{2}} \operatorname{Im}\left(\left[G^{*} \boldsymbol{m}_{d} V^{\dagger} \boldsymbol{m}_{u} G \boldsymbol{m}_{d} V^{T} \boldsymbol{m}_{u}\right]_{33} \frac{P_{0}}{M_{\Phi}^{2}}\right. \\
& +\left[G^{* *} V^{\dagger} G^{\prime} \boldsymbol{m}_{d}^{2} V^{T}\right]_{33} P_{1} \\
& \left.+10\left[G^{* *} V^{\dagger} \boldsymbol{m}_{u} G \boldsymbol{m}_{d} V^{T}+G^{*} \boldsymbol{m}_{d} V^{\dagger} G^{\prime} V^{T} \boldsymbol{m}_{u}\right]_{33} P_{1}\right) .
\end{aligned}
$$

where $g_{w}$ is the weak coupling constant. In Eq. (31), we have simplified the expression by replacing logarithms that arise from the momentum integrals with their order of magnitude values, namely, a factor of 1 in the case of the $G G$ or $G^{\prime} G^{\prime}$ type terms, and a factor of 10 in the case of the mixed $G G^{\prime}$ type terms. The full expressions have been used in the numerical analysis below.

\section{B. Dependence of the asymmetry on quark masses}

An important feature of the expressions for $\epsilon_{\text {wave }}$ and $\epsilon_{\text {vertex }}$ is their dependence on quark masses. Indeed, each of the terms of Eqs. (30) and (31) have a different quark masses dependence, and this determines which contribution dominates the asymmetry generation. The origin of these mass factors can be traced to either the chiral structure of the quark $-\Delta_{u d}$ couplings, or to the symmetry structure of the $G$ and $G^{\prime}$ matrices, as we will outline below.

The way in which the chiral nature of the couplings is related to the mass factors of $\epsilon_{\text {wave }}$ and $\epsilon_{\text {vertex }}$ can be easily understood. If the $G$ coupling to RH quarks is involved, chiral flips are required in order to couple the quarks to the $W$ in the loop diagrams. No such chiral flip is needed in the case of the $G^{\prime}$ coupling to LH quarks. Therefore, the term proportional to $G G$ requires four mass insertions for the 


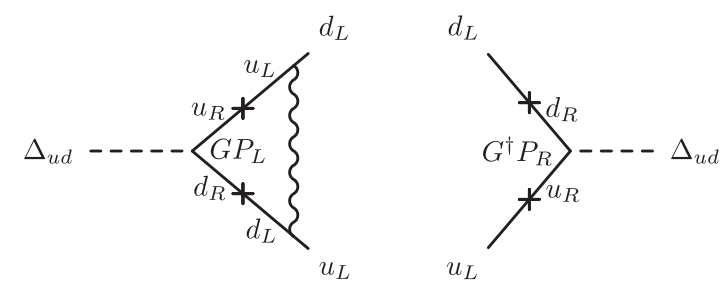

FIG. 5. A diagrammatic explanation of the mass dependence of the vertex correction, in the case where the $\Delta_{u d}$ couples only to RH quarks. The necessity for four mass insertions can be understood as follows: $G$ couples a $\Delta_{u d}$ to two right-handed quarks, hence two mass insertions are required so that the quarks can exchange a $W$-boson. Then, in order for the helicities to correctly match the conjugated tree-level diagram, two more mass insertions are required. The corresponding expression for the asymmetry $\epsilon_{\text {vertex }}$ is found to differ from that [8] and [13], but to agree with that of [17].

vertex correction, ${ }^{6}$ as illustrated in Fig. 5, and two for the wave function correction. The $G G^{\prime}$ cross term requires only two mass insertions for both the vertex and wave function corrections, while the $G^{\prime} G^{\prime}$ term requires no chiral flips for either the wave or vertex topologies.

Inspecting Eq. (30) we see that the wave function corrections appear to require two additional mass quark factors beyond those stipulated above. This occurs because, in the limit that the (down-type) quark in the wave function loop diagram is taken to be massless, the amplitudes all contain a factor of $\left(V^{\dagger} V\right)_{i j}=\delta_{i j}$. This renders the remaining product of coupling factors real. However, by retaining the next order term in a small mass expansion we obtain a $\left(V^{\dagger} m_{d}^{2} V\right)_{i j} \neq \delta_{i j}$ dependence, and hence a product of couplings that, in general, will be complex. ${ }^{7}$

For a similar reason, the $G^{\prime} G^{\prime}$ vertex contribution also has a factor of $m_{d}^{2}$. Again, in the limit of zero quark mass, we would have

$$
\left(G^{* *} V^{\dagger} G^{\prime} V^{T}\right)_{33}=-\left|V_{\mathrm{CKM}}^{*}\left[g^{\prime *}-g^{\prime \dagger}\right]\right|_{3 i}^{2}=\text { Real },
$$

due to the antisymmetry of $g^{\prime}$. (Note also that $V_{\mathrm{CKM}} \rightarrow \mathbb{1}$ in the zero quark mass limit.) Hence we are again required to keep the next order term in quark mass (arising from the cut loop integral) which yields a factor of $m_{d}^{2}$ and results in a coefficient which, in general, is complex.

The quark mass factors in the various contributions to $\epsilon_{\text {wave }}$ and $\epsilon_{\text {vertex }}$ will determine which terms dominate the generation of the baryon asymmetry. Before discussing the magnitudes of the asymmetries produced, we first discuss

\footnotetext{
${ }^{6}$ The necessity of four mass insertions appears to disagree with the expressions of $[8,13]$, but does agree with the work of the same authors in [17].

${ }^{7}$ More generally, it is clear that distinct quarks masses and nontrivial $V_{\mathrm{CKM}}$ mixing are important requirements for successful baryogenesis in this model.
}

the dilution of the baryon asymmetry and its implications for the choices of scalar masses.

\section{Dilution of the baryon asymmetry}

It is insufficient to consider only the $C P$ asymmetry, $\epsilon$, in order to determine the viability of the model as a baryogenesis mechanism, as there may also be a dilution of the final asymmetry. Significant dilution occurs when the scalar $\Phi$ decays far out of equilibrium, such that the entropy released in the decays decreases the baryon to photon ratio. The factor by which the asymmetry is diluted can be approximated by the ratio $T_{d} / M_{\Phi}$, where $T_{d}$ is the temperature of the universe when the decay process occurs. This temperature can be approximated by equating the decay rate $\Gamma$ with the expansion rate $H$. The dilution factor can then be estimated as:

$$
T_{d} / M_{\Phi} \sim 2.7 \times 10^{-4}\left|G+G^{\prime}\right|\left(\frac{M_{\Phi}}{6 M_{\Delta}}\right)^{6}\left(\frac{M_{\mathrm{Pl}}}{M_{\Phi}}\right)^{1 / 2},
$$

where $M_{\mathrm{Pl}}$ is the Planck mass, and the factor of $2.7 \times 10^{-4}$ combines all numerical constants, the color factor and factors from loop integration.

Written in this form it is clear that the dilution factor depends strongly on the ratio $M_{\Phi} / M_{\Delta}$. Therefore, large diquark masses require a correspondingly large mass for the scalar $\Phi$. Furthermore, the allowable scalar masses are restricted by the requirement that the decay occur within a temperature range of:

$$
100 \mathrm{MeV} \lesssim T_{d} \lesssim 100 \mathrm{GeV}
$$

so as not to interfere with big bang nucleosynthesis and to occur at a temperature where sphaleron transitions are suppressed. Given that we have taken $M_{\Delta}=10 \mathrm{TeV}$ in order to satisfy LHC limits on the scalar diquarks, we require $M_{\Phi}$ to be in the $\mathrm{TeV}$ range to avoid excessive dilution. We find that $M_{\Phi} \sim 7 \mathrm{TeV}$ is an appropriate choice that minimizes the dilution while keeping the decay temperature in the required range.

We now examine the size of the $C P$ asymmetry and relevant dilution factors in the context of 3 benchmark coupling scenarios which satisfy our constraints from radiative quark masses and EDMs.

\section{Size of the asymmetry for benchmark scenarios}

To determine the size of the baryon asymmetry in the presence of both the LH and RH couplings, we take the three benchmark scenarios defined by Eq. (26). We generate random complex matrices which satisfy the appropriate symmetries $^{8}$ of $G$ and $g^{\prime}$, where all nonzero elements are of the order of magnitude specified by Eq. (26). We then transform $g^{\prime}$ to $G^{\prime}$ by rotating to the mass basis, and compute

\footnotetext{
${ }^{8}$ See Appendix A for a discussion of the symmetries of $g^{\prime}$.
} 
TABLE IV. Typical sizes of the $C P$ asymmetry in the three benchmark scenarios. The first corresponds to the assumptions of $[8,13]$ in which the $\Delta_{u d}$ couples only to RH quarks, while the second corresponds to the scenario in which the $\Delta_{u d}$ couples only to LH quarks. In the final scenario, the $\Delta_{u d}$ couples to both RH and LH quarks with similar strength. In the case of $G^{\prime}=0$ the asymmetries are smaller than those of [8,17], which adopt a different flavor structure for $G$ and is missing quark mass factors. Again we have assumed the benchmark delta mass of $M_{\Delta}=10 \mathrm{TeV}$. The baryon asymmetry generated by the decay of the scalar $\Phi$ in each scenario is given by multiplying the $C P$ asymmetry and the dilution factor, and must be greater than $10^{-10}$ in order to explain the observed value.

\begin{tabular}{lccc}
\hline \hline & $\epsilon_{\mathrm{wave}}\left(M_{\Phi}=7 \mathrm{TeV}\right)$ & $\epsilon_{\mathrm{vertex}}\left(M_{\Phi}=7 \mathrm{TeV}\right)$ & Dilution factor $\left(M_{\Phi}=7 \mathrm{TeV}\right)$ \\
\hline$G_{\alpha \beta} \sim 1, G_{\alpha \beta}^{\prime}=0$ & $10^{-9}$ & $10^{-14}$ & $10^{-2}$ \\
$G_{\alpha \beta}^{\prime} \sim 1, G_{\alpha \beta}=0$ & $10^{-7}$ & $10^{-8}$ & $10^{-2}$ \\
$G_{\alpha \beta} \sim G_{\alpha \beta}^{\prime} \sim 10^{-3}$ & $10^{-7}$ & $10^{-6}$ & $10^{-5}$ \\
\hline \hline
\end{tabular}

the values $\epsilon_{\text {wave }}$ and $\epsilon_{\text {vertex }}$ according to Eqs. (30) and (31). The typical order of magnitude obtained via this procedure is recorded in Table IV for each contribution to the total asymmetry, again we use a benchmark delta mass of $M_{\Delta}=10 \mathrm{TeV}$.

In the case of the wave function contribution, the term proportional to $G^{\prime} G^{\prime}$ (LH couplings) is weighted by $m_{t}^{2}$ while the term proportion to $G G$ ( $\mathrm{RH}$ couplings) is weighted by $m_{u} m_{t}$. In the latter, $m_{u}$ may be either a $u$-quark or $c$-quark, as $m_{u}=m_{t}$ would lead to an overall real value. We therefore expect that the $G G$ contribution to be suppressed with respect to the $G^{\prime} G^{\prime}$ contribution by a factor of $m_{c} / m_{t} \sim 10^{-2}$.

For the case of the vertex contribution, we instead expect that the $G G$ term is suppressed with respect to the $G^{\prime} G^{\prime}$ term by a factor of $\left(m_{c} m_{t} V_{32}^{\dagger}\right) /\left(6 M_{\Phi}^{2} V_{21}^{\dagger}\right) \sim 10^{-6}$. By comparing the first two lines of Table IV we see that these relative suppression factors are indeed reflected in the values of $\epsilon$ obtained. When $G \sim G^{\prime}$, the $\epsilon$ are dominated by the $G G^{\prime}$ cross terms and the $G^{\prime} G^{\prime}$ contributions.

An important feature of the $\epsilon$ 's is that they are insensitive to the overall magnitude of the couplings $G$ and $G^{\prime}$. This is because both the numerator and denominator (via the terms denoted $\boldsymbol{X}$ ) carry two powers of $G$ or $G^{\prime}$. However, it is not viable to make the couplings arbitrarily small because this would suppress the tree level decay rate, resulting in a large dilution factor and thus a final baryon asymmetry that is too small. This can be seen in equation (33), which shows that the dilution factor depends linearly on the magnitude of the couplings. This implies that the case where both couplings are small, $G \sim G^{\prime} \sim 10^{-3}$, will result in the baryon asymmetry being more heavily diluted, as is reflected in the values shown in Table IV.

From our simple analysis, we are able to conclude that the $G=0, G^{\prime} \sim 1$, benchmark, in which the $\Delta_{u d}$ couples only to LH quarks, provides the most viable baryogenesis scenario and permits $C P$-violating asymmetries up to $\epsilon \sim 10^{-6}$ before any washout or dilution effects are incorporated. The $G \sim 1$, $G^{\prime}=0$ benchmark, in which the $\Delta_{u d}$ couples only to RH quarks, is less viable because the $C P$-violating asymmetries are suppressed by additional factors of light quark mass and/or small CKM matrix elements. The $G \sim G^{\prime}$ benchmark, which features comparable couplings to LH and RH quarks, is disfavoured because the requirement that the magnitudes of the couplings the satisfy the EDM constraints yields too small a tree-level decay rate.

It is important to note that our analysis assumes the coupling matrices have no strong flavor hierarchies. While the adoption of a specific flavor structure could relax our conclusions, a detailed exploration is beyond the scope of this paper. We also note that the $C P$ asymmetry in this model appears at second order in the B-violating coupling. This is in apparent contradiction to the Weinberg-Nanopolous theorem, illustrated in Refs. [30,31], which states that non-zero contributions to the asymmetry must occur at third order in $\mathrm{B}$-violating couplings. The implications of this theorem for the model considered in this paper have previously been considered in Appendix A of Ref. [13], in which a simplified version of the model was shown to permit a nonzero asymmetry at second order in the B-violating coupling. In this toy model the asymmetry is generated by a real scalar, analogous the scalar $\Phi$, while the loop couplings violate flavour but conserve baryon number. Their result showed that the difference in masses of final state flavours prevented the cancellation of contributions to the $C P$ asymmetry at second order. In the full model, this effect of spectral nondegeneracy is realized explicitly through the quark mass differences and implicitly through the non-vanishing off-diagonal elements of the CKM matrix. It should be noted that other exceptions to this theorem have been established in the past, e.g., in [32]. A more detailed analysis of the exceptions to this theorem is deferred to a future work.

\section{CONCLUSIONS}

In this paper we have examined the "postsphaleron baryogenesis" mechanism first introduced in [8]. While the original scenario featured the coupling of scalar diquarks to RH quarks, we extended the model by the addition of couplings to left-handed quarks, $\Delta_{u d} \bar{Q} Q^{c}$, which results in a greatly enhanced baryon asymmetry. 
This occurs simply because baryogenesis proceeds via $W$ loop diagrams, for which contributions involving $\mathrm{LH}$ quarks in general require less quark mass insertions than those involving RH quarks.

This comes at the cost, at least in the scenario in which both LH and RH couplings are present, of induced electric dipole moments of nucleons and atoms and radiative contributions to the quark masses. We find that the electric dipole moments of the neutron and ${ }^{199} \mathrm{Hg}$ provide the most stringent constraints. Under the assumption that there is no significant hierarchy among the elements of LH coupling constant matrix $G^{\prime}$ (and likewise the RH couplings $G$ ) and that the real and imaginary components of these couplings are comparable, the EDM constraints imply that the $G G^{\prime}$ couplings constant products are in the $<10^{-5}-10^{-7}$ range. Hence either $G, G^{\prime}$, or both must be small.

We derived expressions for the self energy and vertex correction contributions to the baryon asymmetry generation, in the presence of couplings to both LH and RH quarks. This allowed up to estimate the relative size of the baryon asymmetry in three benchmark scenarios for which either the $G, G^{\prime}$, or both couplings are nonzero. The most viable scenario was found to be that where only the $G^{\prime}$ couplings to LH quarks are present. The scenario with only $G$ couplings to $\mathrm{RH}$ quarks is disfavored because additional factors of light quark mass suppress the size of the baryon asymmetry generated, while the scenario in which $G$ and $G^{\prime}$ are both nonzero is disfavored by the EDM constraints. Therefore, our new couplings of the diquark scalars to LH quarks is critical for the successful generation of a baryon asymmetry in this model.

\section{ACKNOWLEDGMENTS}

N. F. B., T. C., and M. N. were supported in part by the Australian Research Council. M. J. R. M. was supported in part under U.S. Department of Energy Contract No. DESC0011095. This work was performed in part at the Aspen Center for Physics, which is supported by National Science Foundation Grant No. PHY-1607611.

\section{APPENDIX A: FLAVOR SYMMETRIES AND THE MASS BASIS}

We note that the coefficients $h, f, g$, and $g^{\prime}$ are in general complex $3 \times 3$ matrices. Consequently, the couplings of the $\Delta$ fields to SM fermion bilinears allow for the possibility of $C P$-violation. We may infer further symmetries of these coupling matrices by recalling the sextet representation of $S U(3)$ is symmetric and applying the identity

$$
\bar{\psi} \chi^{c}=\bar{\chi} \psi^{c} .
$$

From here we see that the coupling $h(f)$ which couples a sextet scalar to $\bar{d}_{R} d_{R}^{c}\left(\bar{u}_{R} u_{R}^{c}\right)$ is symmetric in flavor. Expanding the LH coupling of the $\Delta_{u d}$ in its $S U(2)_{L}$ indices and using the above identity as well as that the sextet is symmetric in color allows us to conclude that the matrix $g^{\prime}$ is antisymmetric. There are no such symmetries of the RH coupling matrix $g$. As we will see below rotating to the mass basis obfuscates some of these symmetries.

A symmetric $3 \times 3$ complex matrix contains 6 complex couplings while an antisymmetric $3 \times 3$ complex matrix has three. Thus, in total we have $6+6+3+9=24$ complex couplings $\left(f+h+g^{\prime}+g\right)$ in our model. These are not angles and phases as in the case of the CKM or PMNS matrices as these matrices are not unitary. They are instead a priori unconstrained magnitudes and phases. As there is no remaining freedom to remove further phases by field redefinitions of the quarks we are unable to reduce the size of this set.

As the quantities we calculate in the main text are relevant below EWSB we must rotate to the mass basis. Therefore we rotate the Lagrangian of Eq. (2) to the mass basis. We begin in a flavor diagonal basis where the $d$-quark mass matrix is also diagonal. Then we diagonalize the $u$-quark mass matrix by making the following rotations,

$$
\begin{aligned}
& u_{R, \alpha} \rightarrow\left(T_{u}\right)_{\alpha \beta} u_{R, \beta}^{\prime}, \\
& u_{L, \alpha} \rightarrow\left(S_{u}\right)_{\alpha \beta} u_{L, \beta}^{\prime}=\left(V_{\mathrm{CKM}}\right)_{\alpha \beta} u_{L, \beta}^{\prime},
\end{aligned}
$$

where the primed fields are the fields in the mass basis, and we have noted that starting from a diagonal $d$-quark mass matrix allows us to identify the rotation matrix $S_{u}$ with the CKM matrix. Performing this rotation on the Lagrangian of Eq. (2) we find,

$$
\begin{aligned}
\mathcal{L} \equiv & \frac{\bar{K}}{2} \Delta_{d d}\left(\bar{d}_{R}^{\prime}\right) H\left(d_{R}^{\prime}\right)^{c}+\frac{\bar{K}}{2} \Delta_{u u}\left(\bar{u}_{R}^{\prime}\right) F\left(u_{R}^{\prime}\right)^{c} \\
& +\bar{K} \Delta_{u d}\left(\bar{u}_{R}^{\prime}\right) G\left(d_{R}^{\prime}\right)^{c}+2 \bar{K} \Delta_{u d}\left(\bar{u}_{L}^{\prime}\right) G^{\prime}\left(d_{L}^{\prime}\right)^{c}+\text { H.c.. }
\end{aligned}
$$

Here we have suppressed the flavor indices and introduced the matrices $H, F, G$, and $G^{\prime}$, which are the analogs of the flavor matrices in the mass basis and are given by:

$$
\begin{aligned}
H_{\alpha \beta} & \equiv h_{\alpha \beta} \\
F_{\alpha \beta} & \equiv\left(T_{u}^{\dagger}\right)_{\alpha \sigma} f_{\sigma \rho}\left(T_{u}^{\dagger}\right)_{\rho \beta} \\
G_{\alpha \beta} & \equiv\left(T_{u}^{\dagger}\right)_{\alpha \sigma} g_{\sigma \beta} \\
G_{\alpha \beta}^{\prime} & \equiv\left(V_{\mathrm{CKM}}\right)_{\alpha \sigma}\left[g_{\sigma \beta}^{\prime}-\left(g^{\prime}\right)_{\sigma \beta}^{T}\right] .
\end{aligned}
$$

We note that the asymmetry manifest in the matrix $g^{\prime}$ is no longer present in the matrix $G^{\prime}$. Having rotated to the mass basis we are now free to make calculations in perturbation theory. 


\section{APPENDIX B: EDMS FROM TREE LEVEL EFFECTIVE OPERATORS}

We proceed to integrate out the scalar $\Delta_{u d}$ at tree level by following the procedure outlined in [33]. This procedure is done above EWSB so that we can first match on to the effective operators contained in the review [15]. Assuming that operators of dimension greater than six have negligible effects on the low energy physics allows us to neglect terms of operator mass dimension seven and higher in the expansion. The effective Lagrangian to dimension-six at tree level is given by:

$$
\begin{aligned}
\mathcal{L}_{\mathrm{Eff}}^{\prime}= & -\frac{g_{\alpha \rho} g_{\sigma \beta}^{*}}{M_{\Delta_{u d}}^{2}}\left[\frac{2}{3}\left(Q_{u d}^{(1)}\right)_{\alpha \beta \rho \sigma}+\left(Q_{u d}^{(8)}\right)_{\alpha \beta \rho \sigma}\right] \\
& +\frac{\left[g_{\alpha \rho}^{\prime}-g_{\rho \alpha}^{\prime}\right]\left[\left(g^{\prime}\right)_{\sigma \beta}^{*}-\left(g^{\prime}\right)_{\beta \sigma}^{*}\right]}{4 M_{\Delta_{u d}}^{2}}\left[\left(Q_{q q}^{(1)}\right)_{\alpha \beta \rho \sigma}+\left(Q_{q q}^{(8)}\right)_{\alpha \beta \rho \sigma}\right] \\
& +\frac{g_{\alpha \beta}\left[\left(g^{\prime}\right)_{\sigma \rho}^{*}-\left(g^{\prime}\right)_{\rho \sigma}^{*}\right]}{3 M_{\Delta_{u d}}^{2}}\left[2\left(Q_{q u q d}^{(1)}\right)_{\alpha \rho \beta \sigma}^{\dagger}\right. \\
& \left.+\left(Q_{q u q d}^{(8)}\right)_{\alpha \rho \beta \sigma}^{\dagger}+\text { H.c. }\right] .
\end{aligned}
$$

Where the operators $Q$ are defined as:

$$
\begin{aligned}
& \left(Q_{u d}^{(1)}\right)_{\alpha \beta \rho \sigma}=\left(\bar{u}_{\alpha} \gamma_{\mu} u_{\beta}\right)\left(\bar{d}_{\rho} \gamma^{\mu} d_{\sigma}\right) \\
& \left(Q_{u d}^{(8)}\right)_{\alpha \beta \rho \sigma}=\left(\bar{u}_{\alpha} \gamma_{\mu} T^{A} u_{\beta}\right)\left(\bar{d}_{\rho} \gamma^{\mu} T^{A} d_{\sigma}\right) \\
& \left(Q_{q q}^{(1)}\right)_{\alpha \beta \rho \sigma}=\left(\bar{q}_{\alpha} \gamma_{\mu} q_{\beta}\right)\left(\bar{q}_{\rho} \gamma^{\mu} q_{\sigma}\right) \\
& \left(Q_{q q}^{(8)}\right)_{\alpha \beta \rho \sigma}=\left(\bar{q}_{\alpha} \gamma_{\mu} T^{A} q_{\beta}\right)\left(\bar{q}_{\rho} \gamma^{\mu} T^{A} q_{\sigma}\right)
\end{aligned}
$$

And $Q_{q u q d}^{(1,8)}$ were defined in Eqs. (11) and (12). We note that a linear combination of the $C P$-violating $Q_{q u q d}^{(1,8)}$ is obtained as a result of our application of the following Fierz identities:

$$
\begin{aligned}
\epsilon_{j m} \epsilon_{k n} & =-\tau_{j k}^{J} \tau_{m n}^{J}+\delta_{j n} \delta_{m k} \\
K_{a b}^{A} \bar{K}_{A}^{c d} & =\frac{1}{2}\left(\delta_{a d} \delta_{b c}+\delta_{a c} \delta_{b d}\right) \\
\lambda_{a b}^{I} \lambda_{c d}^{I}= & 2 \delta_{a d} \delta_{b c}-\frac{2}{3} \delta_{a b} \delta_{c d} \\
\left(\bar{\psi}_{1} \sigma^{\mu \nu} P_{L} \psi_{2}\right)\left(\bar{\psi}_{3} \sigma_{\mu \nu} P_{L} \psi_{4}\right)= & -8\left(\bar{\psi}_{1} P_{L} \psi_{4}\right)\left(\bar{\psi}_{3} P_{L} \psi_{2}\right) \\
& -4\left(\bar{\psi}_{1} P_{L} \psi_{2}\right)\left(\bar{\psi}_{3} P_{L} \psi_{4}\right) .
\end{aligned}
$$

It is interesting to note that the asymmetry of the matrix $g^{\prime}$ is manifest in the operator coefficients. Moreover, as only the purely first generation operator contributes to the neutron and ${ }^{199} \mathrm{Hg}$ EDMs we might naïvely think the coefficient vanishes due to this antisymmetry in the flavor indices. However, Eq. (B1) is in the flavor basis and we must now rotate to the mass basis drawing any conclusions about low energy observables.

In the mass basis, we find the $Q_{q u q d}^{(1)}$ term becomes

$$
\begin{aligned}
\mathcal{L}= & -\frac{2}{3 M_{\Delta_{u d}}^{2}} G_{a b}\left(G^{\prime}\right)_{c d}^{\dagger} \\
& \times\left[\left(\bar{u}_{R}^{\prime} u_{L}^{\prime}\right)_{a d}\left(\bar{d}_{R} d_{L}\right)_{b c}+\left(\bar{u}_{R}^{\prime} d_{L}\right)_{a c}\left(\bar{d}_{R} u_{L}^{\prime}\right)_{b d}\right] .
\end{aligned}
$$

A similar result is found for $Q_{q u q d}^{(8)}$ with additional factors of $\lambda^{A}$. Now we see the coefficient does not vanish for purely first generation couplings. We emphasize that these operators are not chirally invariant, in contrast to $Q_{q u q d}^{(1,8)}$ when the latter are constructed solely from first generation quarks. As discussed in the main text, this feature follows from the generation nondiagonal property of the nonvanishing four-quark operators in this scenario.
[1] D. E. Morrissey and M. J. Ramsey-Musolf, Electroweak baryogenesis, New J. Phys. 14, 125003 (2012).

[2] M. Trodden, Electroweak baryogenesis, Rev. Mod. Phys. 71, 1463 (1999).

[3] J. M. Cline, Baryogenesis, arXiv:hep-ph/0609145.

[4] A. Riotto, Theories of baryogenesis, arXiv:hep-ph/9807454.

[5] S. Davidson, E. Nardi, and Y. Nir, Leptogenesis, Phys. Rep. 466, 105 (2008).

[6] W. Buchmuller, R. D. Peccei, and T. Yanagida, Leptogenesis as the origin of matter, Annu. Rev. Nucl. Part. Sci. 55, 311 (2005).

[7] M. Fukugita and T. Yanagida, Baryogenesis without grand unification, Phys. Lett. B 174, 45 (1986).
[8] K. S. Babu, R. N. Mohapatra, and S. Nasri, Post-Sphaleron Baryogenesis, Phys. Rev. Lett. 97, 131301 (2006).

[9] J. M. Arnold, B. Fornal, and M. B. Wise, Simplified models with baryon number violation but no proton decay, Phys. Rev. D 87, 075004 (2013).

[10] I. Baldes, N. F. Bell, and R. R. Volkas, Baryon number violating scalar diquarks at the LHC, Phys. Rev. D 84, 115019 (2011).

[11] P. S. Bhupal Dev and R. N. Mohapatra, TeV scale model for baryon and lepton number violation and resonant baryogenesis, Phys. Rev. D 92, 016007 (2015).

[12] J. P. Bowes, R. Foot, and R. R. Volkas, Electric charge quantization from gauge invariance of a Lagrangian: A 
Catalog of baryon number violating scalar interactions, Phys. Rev. D 54, 6936 (1996).

[13] K. S. Babu, P. S. Bhupal Dev, E. C. F. S. Fortes, and R. N. Mohapatra, Post-Sphaleron baryogenesis and an upper limit on the neutron-antineutron oscillation time, Phys. Rev. D 87, 115019 (2013).

[14] T. Chupp, P. Fierlinger, M. Ramsey-Musolf, and J. Singh, Electric dipole moments of the atoms, molecules, nuclei and particles, Rev. Mod. Phys. 91, 015001 (2019).

[15] J. Engel, M. J. Ramsey-Musolf, and U. van Kolck, Electric dipole moments of nucleons, nuclei, and atoms: The Standard Model and beyond, Prog. Part. Nucl. Phys. 71, 21 (2013).

[16] N. Yamanaka, B. K. Sahoo, N. Yoshinaga, T. Sato, K. Asahi, and B. P. Das, Probing exotic phenomena at the interface of nuclear and particle physics with the electric dipole moments of diamagnetic atoms: A unique window to hadronic and semi-leptonic CP violation, Eur. Phys. J. A 53, 54 (2017).

[17] K. S. Babu, P. S. Bhupal Dev, and R. N. Mohapatra, Neutrino mass hierarchy, neutron-anti-neutron oscillation from baryogenesis, Phys. Rev. D 79, 015017 (2009).

[18] T. Han, I. Lewis, and T. McElmurry, QCD corrections to scalar diquark production at hadron colliders, J. High Energy Phys. 01 (2010) 123.

[19] R. S. Chivukula, P. Ittisamai, K. Mohan, and E. H. Simmons, Color discriminant variable and scalar diquarks at the LHC, Phys. Rev. D 92, 075020 (2015).

[20] A. Alloul, N. D. Christensen, C. Degrande, C. Duhr, and B. Fuks, FeynRules 2.0: A complete toolbox for tree-level phenomenology, Comput. Phys. Commun. 185, 2250 (2014).

[21] T. Hahn, Generating Feynman diagrams and amplitudes with FeynArts 3, Comput. Phys. Commun. 140, 418 (2001).

[22] T. Hahn and M. Perez-Victoria, Automatized one loop calculations in four-dimensions and D-dimensions, Comput. Phys. Commun. 118, 153 (1999).
[23] H. H. Patel, Package-X 2.0: A Mathematica package for the analytic calculation of one-loop integrals, Comput. Phys. Commun. 218, 66 (2017).

[24] W. Dekens and J. de Vries, Renormalization group running of dimension-six sources of parity and time-reversal violation, J. High Energy Phys. 05 (2013) 149.

[25] T. Bhattacharya, V. Cirigliano, R. Gupta, H.-W. Lin, and B. Yoon, Neutron Electric Dipole Moment and Tensor Charges from Lattice QCD, Phys. Rev. Lett. 115, 212002 (2015).

[26] T. Bhattacharya, V. Cirigliano, S. Cohen, R. Gupta, A. Joseph, H.-W. Lin, and B. Yoon (PNDME Collaboration), Iso-vector and Iso-scalar tensor charges of the nucleon from lattice QCD, Phys. Rev. D 92, 094511 (2015).

[27] J. M. Pendlebury et al., Revised experimental upper limit on the electric dipole moment of the neutron, Phys. Rev. D 92, 092003 (2015).

[28] B. Graner, Y. Chen, E. G. Lindahl, and B. R. Heckel, Reduced Limit on the Permanent Electric Dipole Moment of Hg199, Phys. Rev. Lett. 116, 161601 (2016); Erratum, Phys. Rev. Lett. 119, 119901(E) (2017).

[29] A. Bashir, R. Delbourgo, and M. L. Roberts, Multidimensional phase space and sunset diagrams, J. Math. Phys. (N.Y.) 42, 5553 (2001).

[30] D. V. Nanopoulos and S. Weinberg, Mechanisms for cosmological baryon production, Phys. Rev. D 20, 2484 (1979).

[31] E. W. Kolb and S. Wolfram, Baryon number generation in the early Universe, Nucl. Phys. B172, 224 (1980); Erratum, Nucl. Phys. B195, 542(E) (1982).

[32] A. Bhattacharya, R. Gandhi, and S. Mukhopadhyay, Revisiting the implications of CPT and unitarity for baryogenesis and leptogenesis, Phys. Rev. D 89, 116014 (2014).

[33] B. Henning, X. Lu, and H. Murayama, How to use the Standard Model effective field theory, J. High Energy Phys. 01 (2016) 023. 